\title{
Planetesimal formation in turbulent circumstellar disks
}

\author{
David Kirsh ${ }^{1}$ and Ralph Pudritz ${ }^{2}$ \\ ${ }^{1}$ Department of Physics and Astronomy McMaster University, \\ Hamilton ON, L8S 4M1, Canada \\ email: kirshdr@mcmaster.ca \\ ${ }^{2}$ Origins Institute, McMaster University, \\ Hamilton ON, L8S 4M1, Canada \\ email: pudritz@physics.mcmaster.ca
}

\begin{abstract}
Planetesimal formation occurs early in the evolution of a solar system, embedded in the circumstellar gas disk, and it is the crucial first step in planet formation. Their growth is difficult beyond boulder size, and likely proceeds via the accumulation of many rocks in turbulence followed by gravitational collapse - a process we are only beginning to understand. We have performed global simulations of the gas disk with embedded particles in the FLASH code. Particles and gas feel drag based on differential velocities and densities. Grains and boulders of various sizes have been investigated, from micron to $\mathrm{km}$, with the goal of understanding where in the disk large planetesimals will tend to form, what sizes will result, and what size ranges of grains will be preferentially incorporated. We have so far simulated particles vertical settling and radial drift under the influence of gas drag, and their accumulations in turbulent clumps.
\end{abstract}

Keywords. accretion disks, hydrodynamics, turbulence, planetary systems: formation

\section{Introduction}

The planetesimal is the key to planet formation, and yet their growth is not yet completely understood. During the early phases of core accretion, particles grow by colliding and sticking. However, meter-size particles suffer gas drag and fall into the star faster than they can stick and grow. In fact, collisions at this size range generally lead to fragmentation rather than sticking. These two effects prevent growth beyond this size. Rapid growth to larger sizes is a consequence of accumulation and gravitational collapse within disk gas turbulence. Drag on the gas by the particles helps promote this effect, and gets stronger with accumulation - this runaway effect is termed the streaming instability. Johansen et al. 2007 discovered this effect in local periodic shearing box simulations. Here we investigate this process in a global disk model, to determine its efficiency.

\section{The Simulations}

We utilize the FLASH hydrodynamics 3D grid code, which has adaptive mesh refinement that allows us to apply more resolution in the regions that require it (high density or velocity). Given the low-mass disk setup currently used, the gravity of the system is a background (stellar) potential. The disk is a global model, up to one vertical scale height, with a chosen inner radius cut-off. Accretion through the inner edge sets off chaotic spiral waves. There is a background disk corona in the remaining simulation volume. Both consist of adiabatic gas $(\gamma=5 / 3)$ in hydrostatic equilibrium with a central gravitational potential. The corona (Ouyed \& Pudritz 1997) that surrounds the disk is chosen to be spherically stable. The central potential and the corona are Plummer-smoothed (Dyer 


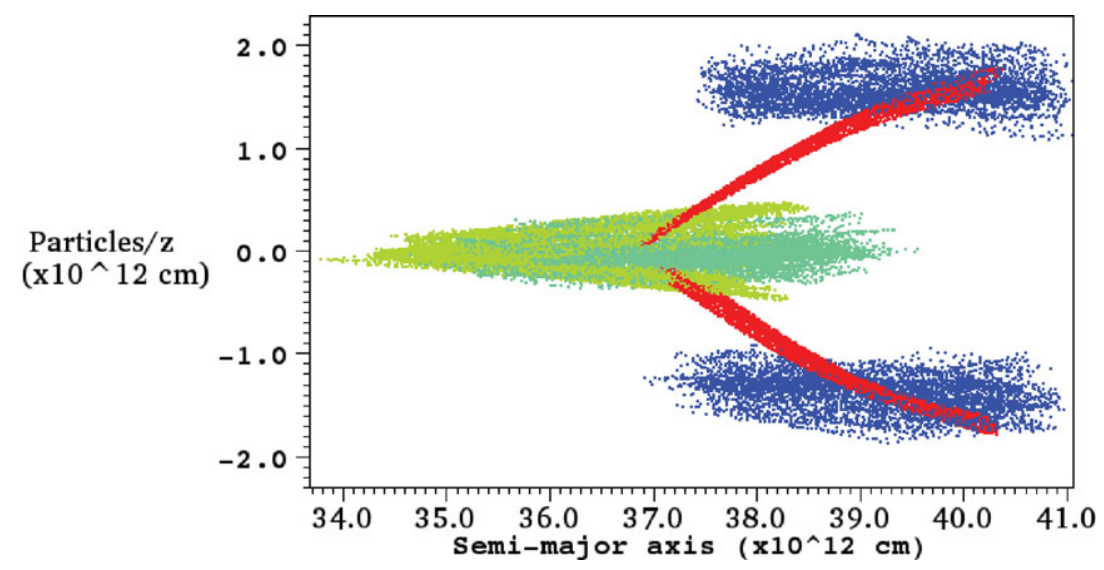

Figure 1. Heights versus semi-major axes for four particle sizes initially placed in two rings of width $0.2 \mathrm{AU}$ at $2.65 \mathrm{AU}(39.6 \mathrm{e} 12 \mathrm{~cm})$, at heights of $\pm 0.4 \mathrm{AU}$ from the midplane, shown after 5 orbits. The $1 \mathrm{~mm}$ particles remain suspended as clumps, only falling a fraction of their height. The $1 \mathrm{~cm}$ particles quickly settle to a thin layer, matching predicted settling rates (Weidenschilling 1977). The $10 \mathrm{~cm}$ particles fall quickly to the midplane but are still oscillating with a relatively small amplitude. These particles, along with the $1 \mathrm{~cm}$ size, show the strongest inward drift. The $1 \mathrm{~m}$ particles oscillate about the midplane, showing only slight reductions in their amplitudes. The curved shape is due to the timescale variation with semi-major axis. Some distortion from the motions of the disk are evident, especially in the smaller sizes.

\& Ip 1993) to prevent a too-sharp gradient. The disk density and pressure decrease vertically from the midplane as an exponential, with the scale height given by the ratio of sound to Keplerian speeds. Pressure support leads to slightly sub-Keplerian gas speed throughout the disk, an effect that the particles do not experience - it is this velocity difference that leads to the particles feeling a headwind drag as they orbit through the slower gas.

The Lagrangian particles are added on top of the grid, interacting via gravity and gas drag determined by the density and velocity of the gas in the cells they occupy, as well as their own physical size, density $\left(\sim 2 \mathrm{~g} / \mathrm{cm}^{3}\right)$ and velocity. They are superparticles, with a mass that represents swarms of many particles. For models where the solid density is roughly $1 / 100$ th the gas density, that mass is divided evenly amongst the particles that are initialized in that cell. They are placed randomly within their cells with Keplerian velocities, and allowed to drift and settle. The drag equations are given by Weidenschilling (1977). Particle drag laws fall into two regimes (Stokes, Epstein) depending on their size compared to the local mean free path of the gas (roughly $10 \mathrm{~cm}$ for our disk). Figure 1 below shows the details of particle settling and drifting in the gas.

We are continuuing to allow the simulations time to run and develop their instabilities. This work will help to show where in the disk planetesimals form, and how efficiently the process occurs.

\section{References}

Dyer, C. C. \& Ip, P. S. S.. 1993, ApJ, 409, 60

Johansen, A., Oishi, J. S., Mac Low, M.-M., Klahr, H., Henning, T., \& Youdin, A. 2007a, Nature, 448,1022

Ouyed, R. \& Pudritz, R. E. 1997, ApJ, 482, 717

Weidenschilling, S. J. 1977, MNRAS, 180, 57 